\title{
Pilot Investigation of Anti-Salmonella Antibodies in Oral Fluids from Salmonella Typhimurium Vaccinated and Unvaccinated Swine Herds
}

\author{
Alessia De Lucia ${ }^{1}$ (), Shaun A. Cawthraw ${ }^{2}$, Richard Piers Smith ${ }^{2}$, Rob Davies ${ }^{2}$, Carlo Bianco ${ }^{3}$, \\ Fabio Ostanello ${ }^{1, *(\mathbb{D})}$ and Francesca Martelli ${ }^{2}$ \\ 1 Department of Veterinary Medical Sciences, School of Agriculture and Veterinary Medicine, \\ via Tolara di Sopra 50, 40064 Ozzano Emilia, Italy; alessia.delucia3@studio.unibo.it \\ 2 Animal and Plant Health Agency, Woodham Lane, New Haw, Addlestone KT15 3NB, UK; \\ shaun.cawthraw@apha.gov.uk (S.A.C.); richard.p.smith@apha.gov.uk (R.P.S.); rob.davies@apha.gov.uk (R.D.); \\ francesca.martelli@apha.gov.uk (F.M.) \\ 3 Animal and Plant Health Agency Lasswade, Pentlands Science Park, Bush Loan, Penicuik, \\ Midlothian EH26 0PZ, UK; carlo.bianco@apha.gov.uk \\ * Correspondence: fabio.ostanello@unibo.it
}

check for updates

Citation: De Lucia, A.; Cawthraw, S.A.; Smith, R.P.; Davies, R.; Bianco, C.; Ostanello, F.; Martelli, F. Pilot Investigation of Anti-Salmonella Antibodies in Oral Fluids from Salmonella Typhimurium Vaccinated and Unvaccinated Swine Herds. Animals 2021, 11, 2408. https:// doi.org/10.3390/ani11082408

Received: 15 July 2021

Accepted: 12 August 2021

Published: 14 August 2021

Publisher's Note: MDPI stays neutral with regard to jurisdictional claims in published maps and institutional affiliations.

Copyright: (c) 2021 by the authors. Licensee MDPI, Basel, Switzerland. This article is an open access article distributed under the terms and conditions of the Creative Commons Attribution (CC BY) license (https:// creativecommons.org/licenses/by/ $4.0 /)$.
Simple Summary: The consumption of pork meat is responsible for a significant number of outbreaks of salmonellosis in people. Surveillance in pig herds is constrained by the cost-effectiveness and efficiency of sampling methods. The last decade has seen significant advances in the routine use of pool samples, including oral fluids (OFs). This study aimed to investigate the OF collected passively via chewed sampling ropes as a potential sample type for assessing anti-Salmonella antibodies in two Salmonella-vaccinated (V) and two non-vaccinated (NV) farrow-to-finish pig farms, comparing the results with the Salmonella shedding of tested animals. Sows in the V farms were vaccinated prior to farrowing. Pooled faecal and OF samples were collected from sows and their offspring. Salmonella was isolated with direct bacteriological methods. A commercial ELISA assay was adapted to detect IgG and IgA antibodies in OF. Overall, a higher Salmonella prevalence was observed in the NV farm and in the offspring (76.3\%) compared to sows (36.4\%). The protocol used to test anti-Salmonella IgA in pig OF samples was found to lack sensitivity and specificity. At herd level, IgG is the most reliable isotype for monitoring Salmonella specific antibody via OF.

Abstract: Oral fluid (OF) can be a simple, cheap and non-invasive alternative to serum or meat juice for the diagnosis and surveillance of important pathogens in pigs. This study was conducted on four Salmonella Typhimurium-positive farrow-to-finish pig farms: two Salmonella-vaccinated (V) and two non-vaccinated (NV). Gilts and sows in the V farms were vaccinated with a live, attenuated vaccine prior to farrowing. Pooled faecal and OF samples were collected from the sows and their offspring. Salmonella was isolated according to ISO6579-1:2017. In parallel, IgG and IgA levels were assessed in OF samples using a commercial ELISA assay. Salmonella was detected in $90.9 \%$ of the pooled faecal samples from the NV farms and in $35.1 \%$ of the pooled faecal samples from the V farms. Overall, a higher prevalence was observed in the pooled faecal samples from the offspring $(76.3 \%)$ compared to the sows (36.4\%). IgG antibodies measured in $\mathrm{V}$ farms are likely to be related to vaccination, as well as exposure to Salmonella field strains. The detection of IgA antibodies in OF was unreliable with the method used. The results of this study show that IgG is the most reliable isotype for monitoring Salmonella-specific antibody immunity in vaccinated/infected animals via OF.

Keywords: antibodies; ELISA; oral fluid; pigs; Salmonella

\section{Introduction}

Non-typhoidal salmonellosis is regarded as one of the most important food-borne zoonotic diseases, causing ill health and high disease-related costs in people $[1,2]$. The 
consumption of pork meat is a major source of human outbreaks [3]. Pigs are susceptible to most Salmonella serotypes and, although Typhimurium and its monophasic variants (mST) are the most common, a large variety of other serotypes are also reported in surveillance studies at farm level [3].

To control the infection in pigs, a combined on-farm approach has been proposed: external and internal biosecurity, control of Salmonella-contaminated feed and vaccination. A live, attenuated vaccine against $S$. Typhimurium in pigs has been developed in Germany (Salmoporc_CEVA Animal Health, Libourne, France) and is currently available in some European countries.

Currently there is no legislation on the control of the Salmonella infection in live pigs. Diagnosis and surveillance for Salmonella in pigs can be carried out on farm or at slaughter using conventional culture methods or serological techniques [4].

Some European countries such Denmark and Germany established their national control programmes to determine the prevalence of Salmonella-positive pig herds on the basis of serological surveillance. Serological monitoring is performed on meat juice collected at the abattoir and tested for Salmonella antibodies using an ELISA. According to the serological status, farms are assigned to three epidemiological levels [5]. Highly infected herds are assigned to level two or three, and farmers are supported by the national governments to reduce the infection load of their herd. Additionally, these farms are subjected to penalty fees to cover the expenses of the special hygienic precautions that have to be taken at the slaughterhouse when pigs from herd level three are slaughtered [6,7]. Farmers are, therefore, motivated to apply better control measures to reduce Salmonella prevalence and avoid the financial consequences [6].

Salmonella surveillance in pig herds is constrained mainly by the cost-effectiveness and efficiency of sampling methods [8]. Disease monitoring often involves blood sampling or environmental samples (floor swabs for Salmonella), which are costly to the farmer due to veterinary fees and labour $[4,8]$. The monitoring of herd health on a regular basis offers accurate diagnostic information and provides options for intervention strategies that can be implemented during the animal's lifetime.

Serological assays using oral fluid (OF) have recently been developed for veterinary diagnostics as OF examination may prove a useful and convenient diagnostic measure of group disease status in pigs [9]. Oral fluid is composed of saliva and a transudate that originates from oral capillaries, particularly gingival crevicular fluid that leaks from the crevices between the teeth and gums [10]. This transudate is a product of the circulatory system and, consequently, contains many of the components found in serum [9]. As such, OF has been described as a diagnostic "mirror of the body", as antibodies from IgA, IgG and IgM classes are all present $[11,12]$. The major antibody class in saliva is secretory $\operatorname{IgA}$ (sIgA) produced by local plasma cells in the salivary gland. In contrast, the major class in crevicular fluid is IgG $[13,14]$. Antibodies of this class are derived from serum, although some IgG antibodies are also locally produced [15]. The presence of local and systemic antibodies in OF suggests they may be suitable for the immunodiagnosis of infectious diseases in live animals. The use of OF has several advantages compared to serum. Sample collection is relatively stress-free for the animals, and cheap and easy to perform, even by unskilled personnel $[16,17]$. Oral fluid offers the possibility of testing pooled samples that facilitates cost-efficient monitoring of the health status of a large population [11,18]. Many serum assays can be optimised to detect antibodies in OF [19] and a number of recent studies have investigated their potential for disease diagnosis in pigs [20-22].

Oral fluid can be obtained using ropes made from a range of natural fibres, such as cotton and hemp, and synthetic fibres, such as polyester and polyamide (water absorbing) or polypropylene and polyethylene (water repellent) [15]. Importantly, the rope material seems to have an impact on the antibody titre obtained and the isotypes of the antibodies collected. Cotton is highly absorbent and reportedly yields higher titres of IgG antibodies compared to other rope types [11,15]. Pig saliva has been used to detect antibodies against 
several specific porcine pathogens and is now routinely used for the virus isolation of important endemic swine pathogens such as PRRSV and PCV2 [21,23].

The objective of this study was to investigate specific anti-Salmonella IgG and IgA antibodies levels in OFs collected from pigs that were vaccinated or not vaccinated against Salmonella Typhimurium, in comparison with the shedding of Salmonella in pooled faecal samples of tested animals.

\section{Materials and Methods}

\subsection{Farms}

The samples were collected from four Salmonella-positive farrow-to-finish indoor farms in the UK, sampled within a previous research project aimed at evaluating the efficacy of a live Salmonella Typhimurium vaccine [24]. The following inclusion criteria were used: (i) indoor breeder-finisher enterprise, (ii) herd size of 100-700 sows, (iii) presence or recent occurrence of Salmonella Typhimurium or mST and (iv) sows free of significant clinical disease that may have affected the efficacy of the vaccine [24]. Two of the randomly selected farms used vaccination against Salmonella (V), while the other two did not (NV). In the V farms, the gilts and sows were vaccinated subcutaneously, at 6 weeks and 3 weeks prior to farrowing with Salmoporc STM (CEVA Animal Health, France). The sows received a booster vaccination three weeks before each farrowing. In all farms, the following three pig categories were sampled: farrowing sows and the offspring weaners (from 4 to 10 weeks) and grower pigs (from 11 to 15 weeks) (Figure 1).

\section{Four farms with Salmonella Typhimurium problems}

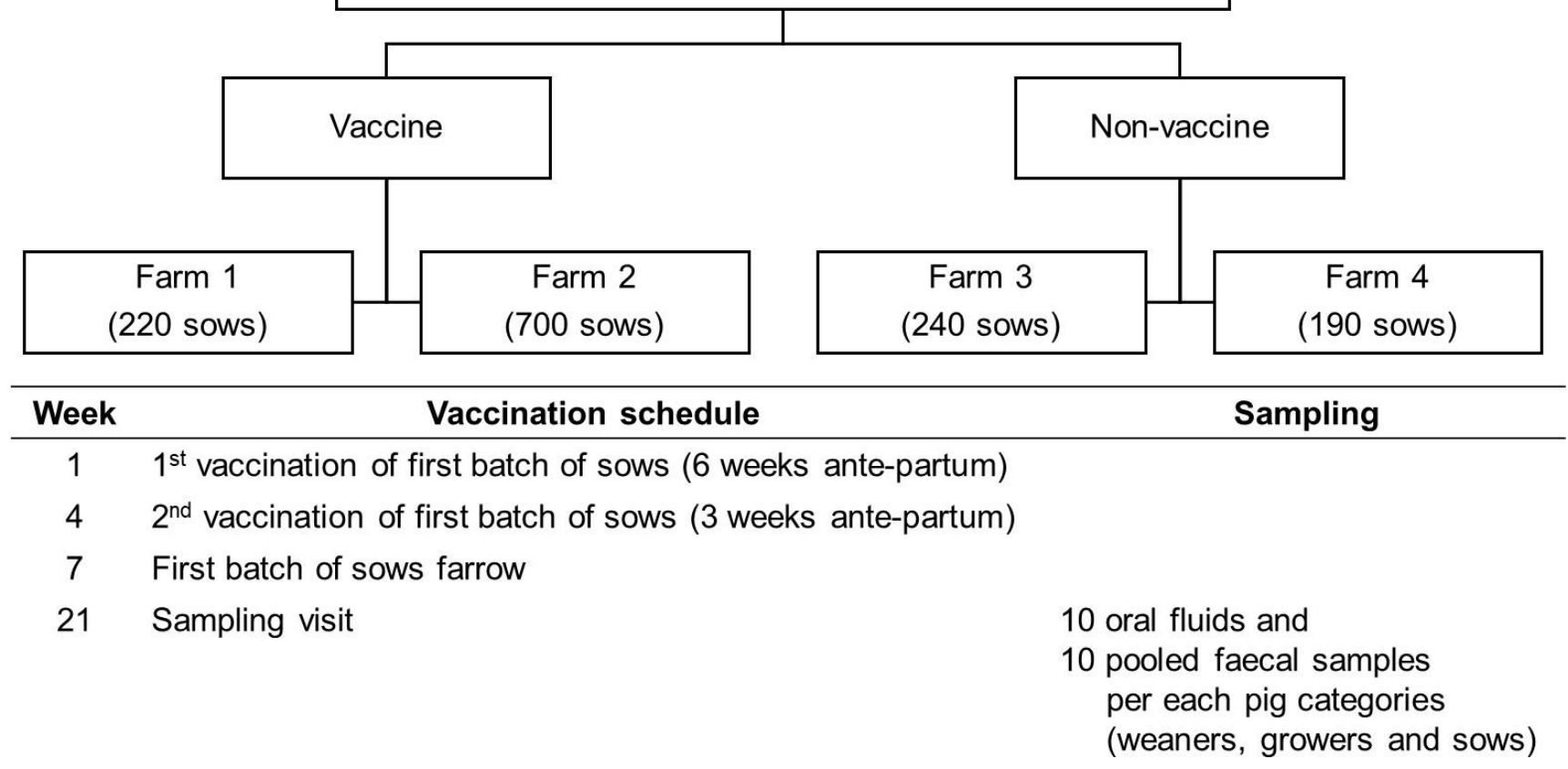

Figure 1. Experimental design: schedule of the vaccination programme and sampling scheme.

One farm visit was carried out to each of the four farms within a three-month period. Sampling visits took place at a point where about half of the progeny on the vaccine farms were estimated to have originated from vaccinated sows. One year after the end of the study, all progenies came from vaccinated sows.

\subsection{Oral Fluid and Pooled Faeces Collection}

In order to see whether a commercial ELISA test validated for serum and meat juice (MJ) was able to accurately detect anti-Salmonella antibodies in OF, samples were obtained 
from different pig categories (farrowing sows, weaners, grower pigs). The sampling scheme was representative of the different production and age categories within farms. Three OFs were collected from first- or second-parity sows, four were collected from third- fourthparity sows and three from the older sows. Regarding the offspring, samples were collected from weaners (about 4-10 weeks old) and growers (about 11-15 weeks old). In each farm and for each pig category, 10 samples of OF were collected. To serve as negative controls in addition to these farm samples, four OF samples were collected from Salmonella-free sows housed in biosecure pens at the Animal and Plant Health Agency (APHA), UK.

For OF sample collection, 50-centimeter-long cotton ropes were placed at pig shoulder height and left in pens of 25-30 pigs for 30 to $60 \mathrm{~min}$, in order to allow approximately $75 \%$ of animals in the pen to chew the rope [23]. When group sizes were larger, one rope for each multiple of 30 pigs was hung in different areas within the same pen. Where there were multiple ropes in a single pen, each rope was treated as single sample rather than pooled, as pooling may influence diagnostic results. Ropes were then placed in individual plastic bags with minimal handling to avoid cross-contamination, transported chilled to the laboratory in less than four hours and refrigerated $\left(+4^{\circ} \mathrm{C}\right)$ overnight. The following day, OFs were extracted by squeezing the ropes and collected into tubes. All samples were centrifuged $(4650 \times g$ per $10 \mathrm{~min})$ and the supernatants stored in aliquots at $-80^{\circ} \mathrm{C}$.

From each pig category, approximately $25 \mathrm{~g}$ pooled faecal samples were taken from the floor with a fabric hand swab and placed directly into $225 \mathrm{~mL}$ of buffered peptone water (BPW; Merck, Darmstadt, Germany, 1.07228.0500) [25].

\subsection{Bacteriological Analyses of Salmonella Prevalence in Pooled Faecal Samples}

Salmonella was isolated according to a modification of ISO6579-1:2017. Briefly, all inoculated BPW samples were incubated at $37 \pm 1{ }^{\circ} \mathrm{C}$ for $16-20 \mathrm{~h}$ and, subsequently, $0.1 \mathrm{~mL}$ of each was inoculated onto modified semi-solid Rappaport-Vassiliadis (MSRV; Mast Group, Bootle, UK, DM440D, with addition of $1 \mathrm{mg} / \mathrm{mL}$ of novobiocin, Sigma-Aldrich, Darmstadt, Germany, N1628) enrichment agar and incubated at $41.5 \pm 1{ }^{\circ} \mathrm{C}$ for $24 \pm 3 \mathrm{~h}$. Growth on MSRV was sub-cultured onto Rambach agar (Merck, Darmstadt, Germany, 1.07500.0002), which was incubated at $37 \pm 1{ }^{\circ} \mathrm{C}$ for $24 \pm 3 \mathrm{~h}$. Serotypes were determined for all isolates according to the White-Kauffmann-Le Minor scheme [26].

\subsection{Detection of Anti-Salmonella IgG and IgA in OF samples}

Anti-Salmonella IgG antibodies present in the OF were measured using the IDEXX Swine Salmonella Ab Test (IDEXX Laboratories, Westbrook, ME, USA), which has been validated for serum and MJ but not for OF. A previous study has shown that under field conditions and at different cut-points of sera optical density (OD), this ELISA kit has a sensitivity of $29-53 \%$ and specificity of $72-93 \%$ when compared with faecal culture results [27]. Always according to the cut-points of OD values, results of MJ examination with this ELISA kit have a relative sensitivity of $53-92 \%$ and relative specificity of $64-84 \%$ when results from serum ELISA were used as gold standard [28]. The manufacturer's protocol was followed except that the OF samples were tested (diluted 1:1 in dilution buffer) instead of serum or MJ. With these changes, previous results indicate that saliva samples had a sensitivity and specificity of 86 and $80 \%$, respectively, when compared with ELISA results obtained from individual serum samples [29].

The IDEXX ELISA plates were also used to detect Salmonella-specific IgA in OF samples. The protocol outlined above was followed except that the kit conjugate was replaced with an anti-porcine IgA HRP conjugate (Abcam, Cambridge, UK), used at 1:10,000 dilution. The positive and negative kit controls and OF collected from four Salmonella-free sows housed in biosecure pens at APHA were included on each plate. In order to account for variation within the assay, all samples were tested in duplicate, and the coefficient of variation (CV) was calculated. 


\subsection{Statistical Analyses}

The Kolmogorov-Smirnov test (K-S) for goodness of fit was used to verify normality of the anti-Salmonella IgG and IgA OD values. According to the K-S test results, the Mann-Whitney (M-W) U-test was used to compare anti-Salmonella IgG and IgA OD values between $\mathrm{V}$ and $\mathrm{NV}$ farms and between production and age categories. Fisher's chi-square test was used to compare prevalence of Salmonella isolation in pooled faecal samples between farm categories $(\mathrm{V}, \mathrm{NV})$ and between production categories (sows and offspring). To measure how strongly Salmonella positivity in pooled faecal samples was associated with the absence of vaccination, odds ratio (OR) and 95\% confidence intervals (95\%CI) were calculated. To assess a possible correlation between Salmonella prevalence in pooled faecal samples and anti-Salmonella IgG and IgA OD values, the nonparametric Spearman's rho correlation coefficient was calculated.

Statistical significance was set at $p \leq 0.05$. All statistical analyses were performed using the software SPSS 25.0.0 (IBM SPSS Statistics, Armonk, NY, USA).

\section{Results}

\subsection{Detection of Anti-Salmonella IgG and IgA in OF Samples}

A total of 120 OF samples were collected, but 21 were discarded as they were faecally contaminated and a further 18 were discarded as the ropes had not been chewed. The 81 OF samples that were tested originated from V farms (37) and NV farms (44) (Table 1).

Table 1. Number of oral fluid (OF) samples examined from vaccinated (V) and non-vaccinated (NV) pigs of three pig categories.

\begin{tabular}{cccc}
\hline Pig Category & Farms 1 and 2 (V) & Farms 3 and 4 (NV) & Total \\
\hline Weaners & 13 & 16 & 29 \\
Growers & 11 & 19 & 30 \\
Sows & 13 & 9 & 22 \\
Total OF samples & 37 & 44 & 81 \\
\hline
\end{tabular}

The volume of $\mathrm{OF}$ obtained from the ropes ranged from 2-10 $\mathrm{mL}$ (except for one sample that was only tested for anti-Salmonella IgA). The intra-assay CV (CV intra-assay) was calculated as the average of the individual CVs. The CV was between 0 and $20.8 \%$ ( $\mathrm{CV}$ intra-assay: $4.15 \%$ ) and between 0 and 30.9\% ( $\mathrm{CV}$ intra-assay: $7.3 \%$ ) for IgG and IgA, respectively. Usually, a $\mathrm{CV}_{\text {intra-assay }}$ of $10 \%$ or less is considered satisfactory [30].

The median, minimum and maximum ELISA OD values detected in OF are shown in Table 2.

Considering all the pig categories, the assays for IgG detected a significant higher anti-Salmonella IgG OD value in the OF samples of the $\mathrm{V}$ farms ( $\mathrm{M}-\mathrm{W} \mathrm{U}=470.5 ; p=0.002)$ (Figure 2). In contrast, there was no significant difference between the anti-Salmonella IgA OD values between the $\mathrm{V}$ and $\mathrm{NV}$ farms ( $\mathrm{M}-\mathrm{W} \mathrm{U}=799.5 ; p=0.887$ ) (Figure 2).

In both the $\mathrm{V}$ and $\mathrm{NV}$ farms, there was no significant difference between the antiSalmonella IgG in the OF of sows and their offspring (M-W U = 131.0; $p=0.558$ and $\mathrm{M}-\mathrm{W}$ $\mathrm{U}=108.0 ; p=0.156$, respectively) (Figure 3).

Only the sows in the $\mathrm{V}$ farms had significantly higher IgA levels than their offspring $(\mathrm{M}-\mathrm{W} \mathrm{U}=83.0 ; p=0.02)$ (Table 2).

\subsection{Bacteriological Results of Salmonella Prevalence from Pooled Faeces}

Eighty-one pooled faecal samples were collected using a hand-held gauze; 37 from the $\mathrm{V}$ and 44 from the NV farms. Details from the 81 bacteriological faecal pool samples examined are presented in Table 3. 
Table 2. Median ELISA OD values (min-max) for anti-Salmonella IgA and IgG antibodies detected using IDEXX ELISA in oral fluid (OF) samples from four farms.

\begin{tabular}{|c|c|c|c|c|c|c|c|c|c|c|c|}
\hline Isotype & $\begin{array}{c}\text { Farm } \\
\text { Category }\end{array}$ & Farm id. & Sows & & Weaners & & Grower & & $\begin{array}{c}\text { Offspring } \\
\text { (Weaners and Growers) }\end{array}$ & All Anim & \\
\hline \multirow{7}{*}{$\operatorname{IgG}$} & \multirow[b]{2}{*}{$\mathrm{V}$} & 1 & $0.13(0.07-0.82)$ & $(4)^{a}$ & $0.30(0.19-0.34)$ & (7) & $0.23(0.17-0.48)$ & $(8)$ & $0.26(0.17-0.48)$ & $0.24(0.07-0.82)$ & (19) \\
\hline & & 2 & $0.39(0.07-2.49)$ & (9) & $0.18(0.03-0.32)$ & (6) & $0.09(0.08-0.09)$ & $(2)^{b}$ & $0.14(0.03-0.32)$ & $0.22(0.03-2.49)$ & (17) \\
\hline & \multirow{2}{*}{ NV } & 3 & $0.10(0.09-0.14)$ & (6) & $0.11(0.09-0.28)$ & $(7)$ & $0.11(0.09-0.57)$ & (10) & $0.11(0.09-0.57)$ & $0.11(0.09-0.57)$ & (23) \\
\hline & & 4 & $0.17(0.08-0.21)$ & (3) & $0.14(0.11-0.37)$ & (9) & $0.13(0.10-0.34)$ & (9) & $0.14(0.10-0.37)$ & $0.14(0.08-0.37)$ & (21) \\
\hline & $\mathrm{V}$ & $1+2$ & $0.22(0.07-2.49)$ & (13) & $0.23(0.03-0.34)$ & (13) & $0.22(0.08-0.48)$ & $(10)$ & $0.23(0.03-0.48)$ & $0.22(0.03-2.49)$ & (36) \\
\hline & NV & $3+4$ & $0.11(0.08-0.21)$ & (9) & $0.12(0.09-0.37)$ & (16) & $0.13(0.09-0.57)$ & (19) & $0.12(0.09-0.57)$ & $0.12(0.08-0.57)$ & $(44)$ \\
\hline & $\mathrm{V}+\mathrm{NV}$ & all farms & $0.16(0.07-2.49)$ & $(22)$ & $0.15(0.03-0.37)$ & (29) & $0.17(0.08-0.57)$ & (29) & $0.16(0.03-0.57)$ & $0.16(0.03-2.49)$ & $(80)$ \\
\hline \multirow{7}{*}{$\operatorname{Ig} A$} & \multirow{2}{*}{$\mathrm{V}$} & 1 & $0.32(0.31-0.66)$ & (4) & $0.28(0.09-0.51)$ & (7) & $0.44(0.21-1.83)$ & $(8)$ & $0.35(0.09-1.83)$ & $0.33(0.09-1.83)$ & (19) \\
\hline & & 2 & $0.53(0.06-1.38)$ & (9) & $0.08(0.06-0.14)$ & (6) & $0.12(0.10-0.20)$ & (3) & $0.10(0.06-0.20)$ & $0.15(0.06-1.38)$ & (18) \\
\hline & \multirow[b]{2}{*}{ NV } & 3 & $0.28(0.20-0.42)$ & (6) & $0.07(0.05-0.20)$ & (7) & $0.16(0.08-0.51)$ & (10) & $0.13(0.05-0.51)$ & $0.18(0.05-0.51)$ & (23) \\
\hline & & 4 & $0.62(0.17-1.08)$ & (3) & $0.35(0.20-0.47)$ & (9) & $0.53(0.25-1.22)$ & (9) & $0.46(0.20-1.22)$ & $0.47(0.17-1.22)$ & (21) \\
\hline & $\mathrm{V}$ & $1+2$ & $0.46(0.06-1.38)$ & (13) & $0.14(0.06-0.51)$ & (13) & $0.43(0.10-1.83)$ & (11) & $0.22(0.06-1.83)$ & $0.31(0.06-1.83)$ & (37) \\
\hline & NV & $3+4$ & $0.30(0.17-1.08)$ & (9) & $0.23(0.05-0.47)$ & (16) & $0.47(0.08-1.22)$ & (19) & $0.27(0.05-1.22)$ & $0.29(0.05-1.22)$ & (44) \\
\hline & $\mathrm{V}+\mathrm{NV}$ & all farms & $0.35(0.06-1.38)$ & $(22)$ & $0.20(0.05-0.51)$ & $(29)$ & $0.43(0.08-1.83)$ & (30) & $0.25(0.05-1.83)$ & $0.30(0.05-1.83)$ & (81) \\
\hline $\operatorname{IgA}$ & \multicolumn{2}{|c|}{ Negative control $^{c}$} & $0.54(0.26-1.12)$ & (4) & - & - & - & - & & & \\
\hline
\end{tabular}

\footnotetext{
${ }^{a}$ number of examined rope; ${ }^{b}$ One OF sample was tested only for anti-Salmonella IgA; ${ }^{c}$ four Salmonella-free sows housed in biosecure pens at the Animal and Plant Health Agency, UK.
} 


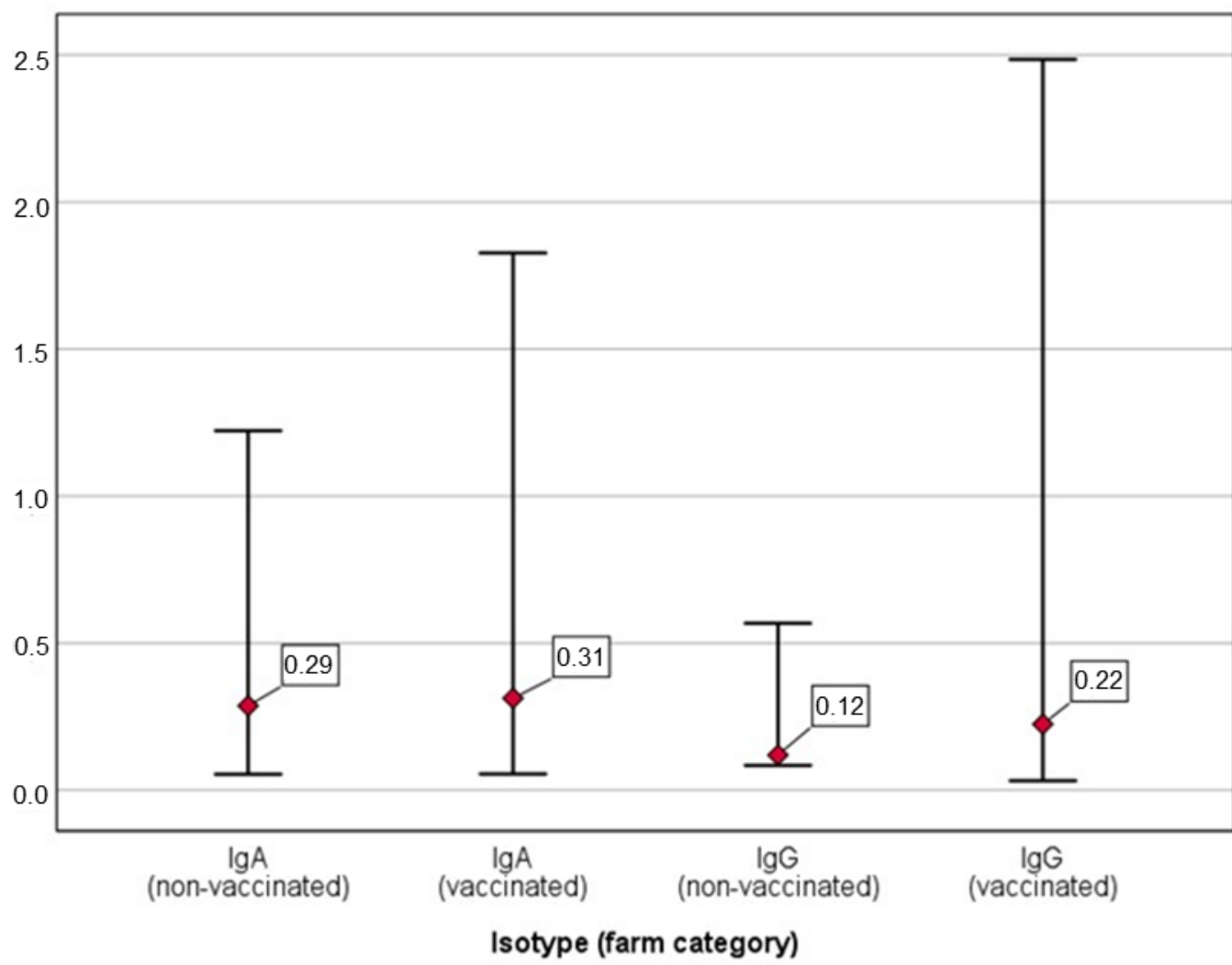

ELISA OD

I $\max$

$\checkmark$ Median

Figure 2. IgG and IgA ELISA OD value in the oral fluid samples from vaccinated and non-vaccinated farms (all pig categories).

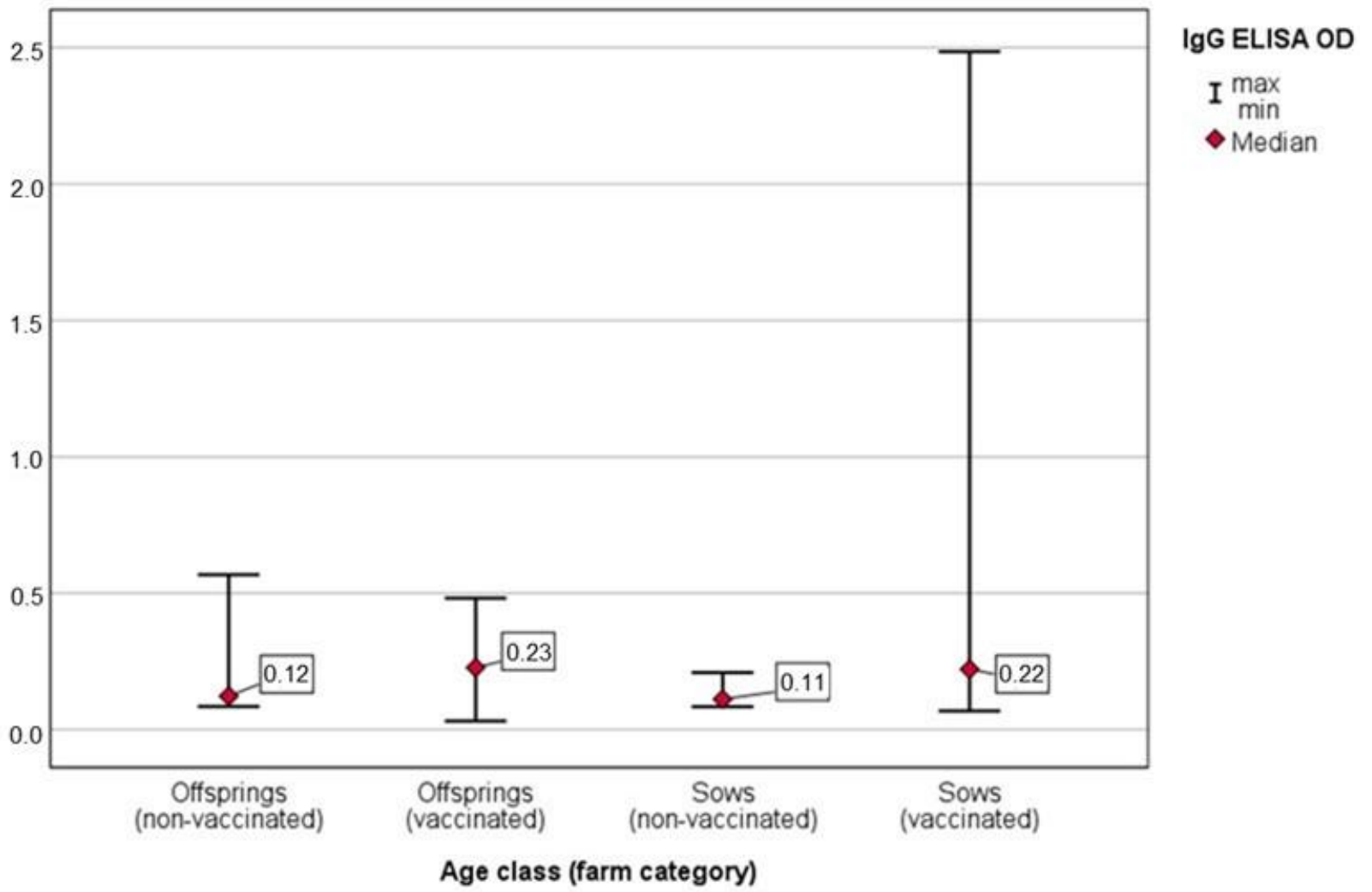

Figure 3. IgG ELISA OD value in the oral fluid samples from sows and offspring in vaccinated and non-vaccinated farms. 
Table 3. Bacteriological results of samples collected from V and NV farms.

\begin{tabular}{|c|c|c|c|c|c|c|}
\hline \multirow[t]{3}{*}{$\begin{array}{c}\text { Farm } \\
\text { Category }\end{array}$} & \multirow{3}{*}{$\begin{array}{c}\text { Pig Category } \\
\text { Weaners }\end{array}$} & \multirow{3}{*}{$\begin{array}{c}\text { Farm } \\
1 \\
2\end{array}$} & \multicolumn{2}{|c|}{$\begin{array}{l}\text { No. of Positive/Examined } \\
(\%)\end{array}$} & \multirow{3}{*}{$\begin{array}{c}p \\
<0.01\end{array}$} & \multirow{3}{*}{$\begin{array}{c}\text { Serotype } \\
4,5,12: \mathrm{i}:-(7) \\
-\end{array}$} \\
\hline & & & $7 / 7$ & $(100)$ & & \\
\hline & & & $0 / 6$ & $(0.0)$ & & \\
\hline \multirow{8}{*}{$\mathrm{V}$} & \multirow{2}{*}{ Growers } & 1 & $4 / 8$ & $(50.0)$ & \multirow[t]{2}{*}{0.24} & 4,5,12:i:- (4) \\
\hline & & 2 & $0 / 3$ & $(0.0)$ & & - \\
\hline & \multirow{2}{*}{ Offspring a } & 1 & $11 / 15$ & $(73.3)$ & \multirow{2}{*}{$<0.001$} & 4,5,12:i:- (11) \\
\hline & & 2 & $0 / 9$ & $(0.0)$ & & - \\
\hline & \multirow{2}{*}{ Sows } & 1 & $0 / 4$ & $(0.0)$ & \multirow[b]{2}{*}{1} & - \\
\hline & & 2 & $2 / 9$ & $(22.2)$ & & Typhimurium (2) \\
\hline & \multirow{2}{*}{ All pig categories } & 1 & $11 / 19$ & $(57.9)$ & \multirow{2}{*}{$<0.01$} & 4,5,12:i:- (11) \\
\hline & & 2 & $2 / 18$ & (11.1) & & Typhimurium (2) \\
\hline \multirow{10}{*}{ NV } & \multirow[b]{2}{*}{ Weaners } & 3 & $7 / 7$ & $(100)$ & \multirow[b]{2}{*}{1} & Kedougou (7) \\
\hline & & 4 & $9 / 9$ & $(100)$ & & 4,5,12:i:- (9) \\
\hline & \multirow{2}{*}{ Growers } & 3 & $10 / 10$ & $(100)$ & \multirow{2}{*}{0.47} & Kedougou (10) \\
\hline & & 4 & $8 / 9$ & (88.9) & & 4,5,12:i:- (8) \\
\hline & \multirow{2}{*}{ Offspring } & 3 & $17 / 17$ & $(100)$ & \multirow{2}{*}{1} & Kedougou (17) \\
\hline & & 4 & $17 / 18$ & $(94.4)$ & & 4,5,12:i:- (17) \\
\hline & \multirow{2}{*}{ Sows } & 3 & $3 / 6$ & $(50.0)$ & \multirow{2}{*}{0.46} & Kedougou (1); 4,5,12:i:- (2) \\
\hline & & 4 & $3 / 3$ & (100) & & 4,5,12:i:- (3) \\
\hline & \multirow{2}{*}{ All pig categories } & 3 & $20 / 23$ & $(87.0)$ & \multirow{2}{*}{0.61} & Kedougou (18); 4,5,12:i:- (2) \\
\hline & & 4 & $20 / 21$ & $(95.2)$ & & 4,5,12:i:- $(20)$ \\
\hline $\mathrm{V}$ & Weaners & 1 and 2 & $7 / 13$ & $(53.8)$ & \multirow{2}{*}{$<0.01$} & $4,5,12: \mathrm{i}:-(7)$ \\
\hline NV & Weaners & 3 and 4 & $16 / 16$ & $(100)$ & & Kedougou (7); 4,5,12:i:- (9) \\
\hline $\mathrm{V}$ & Growers & 1 and 2 & $4 / 11$ & $(36.4)$ & \multirow{2}{*}{$<0.01$} & $4,5,12: \mathrm{i}:-(4)$ \\
\hline NV & Growers & 3 and 4 & $18 / 19$ & $(94.7)$ & & Kedougou (10); 4,5,12:i:- (8) \\
\hline $\mathrm{V}$ & Offspring & 1 and 2 & $11 / 24$ & $(45.8)$ & \multirow{2}{*}{$<0.001$} & 4,5,12:i:- (11) \\
\hline NV & Offspring & 3 and 4 & $34 / 35$ & $(97.1)$ & & Kedougou (17); 4,5,12:i:- (17) \\
\hline $\mathrm{V}$ & Sows & 1 and 2 & $2 / 13$ & $(15.4)$ & \multirow{2}{*}{$<0.05$} & Typhimurium (2) \\
\hline NV & Sows & 3 and 4 & $6 / 9$ & $(66.7)$ & & Kedougou (1); 4,5,12:i:- (5) \\
\hline $\mathrm{V}$ & All pig categories & 1 and 2 & $13 / 37$ & $(35.1)$ & $<0.001$ & Typhimurium (2); 4,5,12:i:- (11) \\
\hline NV & All pig categories & 3 and 4 & $40 / 44$ & $(90.9)$ & & Kedougou (18); 4,5,12:i:- (22) \\
\hline
\end{tabular}

${ }^{a}$ Weaners and Growers.

In the two V farms, from the 37 faecal samples taken, Salmonella was recovered from 13 samples $(35.1 \%)$. Of these, two were S. Typhimurium and 11 were mST. From the 44 faecal samples collected in the NV farms, Salmonella was recovered from 40 samples (90.9\%). mST was also isolated from both of these farms (22 of 40 positive samples), although in one of these farms, $S$. Kedougou was more prevalent (18 of 40) (Table 3).

In the NV farms, there was a significantly higher $(p<0.001)$ Salmonella prevalence of pooled faecal samples than in the V farms (90.9\% vs. 35.1\%) (Table 3). Single-factor-analyses revealed statistically significant associations between Salmonella positivity in pooled faecal samples and the absence of vaccine use (OR: 18.5; 95\%CI 5.4-63.1).

Similarly, in the NV farms, the prevalence of both sows and offspring was significantly higher than in the $\mathrm{V}$ farms ( $p<0.05$ and $p<0.001$, respectively), (Table 3).

A significant $(p=0.001)$ higher Salmonella prevalence was detected in samples collected from the offspring $(45 / 59 ; 76.3 \%)$ when compared to the sows $(8 / 22 ; 36.4 \%)$. 
In the NV farms, the Salmonella prevalence of both sows and offspring and of all the pig categories was not significantly different $(p>0.05)$ between the two farms (3 and 4$)$, (Table 3).

In the $\mathrm{V}$ farms, a statistically significant difference in the Salmonella prevalence in samples collected from the offspring $(p<0.001)$ and from all the pig categories $(p<0.01)$ was observed between the two farms (1 and 2$)$. On the contrary, the prevalence in sows was not significantly different $(p>0.05)$.

3.3. Correlation between Salmonella Prevalence in Pooled Faecal Samples and Anti-Salmonella IgG and IgA OD Values

In the $\mathrm{V}$ farms, when the median values of anti-Salmonella IgG and IgA were compared with Salmonella prevalence using the Spearman's rho coefficient, no correlation was observed ( $r h o: 0.121 ; p=0.484$ and $r h o: 0.268 ; p=0.108$, respectively).

On the contrary, in the NV farms, a positive correlation was observed between the median of the anti-Salmonella IgG and IgA level and Salmonella prevalence (rho: 0.358; $p=0.017$ and rho: $0.0632 ; p<0.001$, respectively).

\section{Discussion}

This study aimed to investigate the levels of anti-Salmonella IgG and IgA antibodies in OF in pigs from herds vaccinated or not vaccinated with a live, attenuated Salmonella vaccine. In addition, the bacteriological status of pooled faecal samples was also determined.

Antibody levels were determined using a commercially available ELISA (IDEXX Swine Salmonella Ab Test) that has been validated for the detection of Salmonella antibodies in porcine serum and MJ. The ELISA kit used in this study has also been used to test porcine OF for anti-Salmonella antibodies in a previous study [21,31]. Recently, the correlation of anti-Salmonella antibodies between serum, OF and saliva samples collected from pigs was evaluated using this commercial kit [29]. The assay screens for the presence of antibodies to the most commonly occurring Salmonella serogroups (B, C1, D) in pigs. S. Typhimurium and its variants, which belong to serogroup $B$, were recovered from the samples from each study farm. However, a group $G$ serovar, $S$. Kedougou, was also isolated from one of the NV farms, more frequently than $S$. Typhimurium. Antibodies to this serovar may not be detected by the assay used in this study [32]. Furthermore, $S$. Kedougou is not normally considered an invasive serovar and, therefore, may induce a more moderate systemic antibody response. It has been suggested that serological testing may have a limited role in monitoring infection by non-invasive Salmonella serotypes [33]. However, anti-Salmonella antibodies were detected in the OF collected in this farm, suggesting that those animals had a mixed infection with $S$. Typhimurium and $S$. Kedougou. Salmonella co-infections are not rare in pig herds [34], but for this study, serotyping was performed on only one colony from each positive faecal sample; therefore, it is likely that only the predominant serovar was detected.

IgG is the most abundant isotype in blood and extracellular fluid, while IgA is predominant at mucosal surfaces and in secretions, including saliva. IgA antibodies are a first line of defence against pathogens, preventing the attachment of bacteria or toxins to epithelial cells [35]. Our results relating to anti-Salmonella IgG antibodies in OFs reflect observations from similar studies using serum as diagnostic material [36]. Specific salivary IgG antibodies have been previously reported as potential indicators of enteric infections [37,38], showing that OF anti-Salmonella IgG antibodies assessment may represent a simple, cheap and non-invasive alternative to serum or MJ. In this study, a positive correlation was observed between the anti-Salmonella IgA level and Salmonella prevalence in the NV farms. However, anti-Salmonella IgA detection was found to be unreliable and non-specific. Indeed, the IDEXX kit detected high anti-Salmonella IgA OD values in the OF of the four Salmonella-negative sows used as controls, suggesting a false positive and non-specific binding (Table 2). The use of cotton ropes seems to be appropriate and recommended to yield higher amounts of IgG. It has been reported that cotton fibre can result in lower IgA concentrations when compared with synthetic fibres [11]. However, results 
from a recent study showed strong anti-Salmonella IgA responses in OF samples collected with cotton rope [31]. There are key differences between this study and ours. The study by Atkinson et al. [31] was a controlled challenge study conducted under experimental conditions that differ considerably to the field conditions of our study. Controlled experimental conditions are presumably less stressful than those found in the intensive pig production units studied in our trial. The animals in a study conducted by Atkinson et al. [31] underwent a single experimental infection and were tested within a short time frame from the infection, whilst the pigs in our investigation were naturally and chronically infected by Salmonella. Furthermore, the specific, strong IgA responses were only detected using an in-house ELISA, using a whole cell antigen preparation made from the challenge strain.

The results from direct diagnostic methods (bacteriology) and indirect diagnostic methods (serology) may not necessarily correlate. The culture of Salmonella indicates true infection and transmission, whereas positive serology may also indicate latent infection within the herd or previous infection [39]. In our study, a lower Salmonella prevalence was detected in the V farms, in particular for the vaccinated sows (Table 3). However, an important variability was observed within the two farm categories (Figures 2 and 3), especially within the V farms. In V farm 1, a lower Salmonella prevalence was found in sows compared with their offspring, as would be expected, while in V farm 2, the opposite was observed, with a higher prevalence found in the vaccinated sows (Table 3). There are several plausible explanations for the variability in the vaccine effect in terms of prevalence in the pig categories. Each pig herd is unique regarding biosecurity measures, management, location, facilities, host susceptibility and other influential factors, which can lead to the large variability within the two farm categories, V and NV [24]. Despite that, the odds ratio of Salmonella-positive samples was significantly higher in the NV compared to the V farms.

In the NV farms, a positive correlation was observed between the median of the antiSalmonella IgG level and the Salmonella prevalence. The high Salmonella burden with high antibody levels in the offspring (in comparison with the sows) suggested that the antibody response was related to infection with field Salmonella strains. Differently, the significantly $(p<0.001)$ lower Salmonella prevalence in the V farms, as would be expected, is likely related to the higher antibody levels detected in the OFs of vaccinated sows, which decreases in their offspring due to the lacking vaccination booster (Figure 3). As already showed by a recent study, the high level of IgG antibodies in the OFs of vaccinated sows may arise from important systemic and mucosal humoral immune responses to anti-Salmonella vaccination [40]. Differences in IgG antibody OD values were also observed in the pig categories of the V and NV farms (Table 2 and Figure 3). In one of the V farms (farm 1), vaccine-induced responses are presumably, at least partly, responsible for the higher levels of IgG antibodies in the OF samples seen in the vaccinated sows compared to those in their non-vaccinated offspring (Table 2). In contrast, on the NV farms, higher IgG OD values were observed in the offspring (in comparison with the sows). This could indicate higher levels of current/recent infection in the younger animals, as supported by the bacteriological results (Table 3). It is important to consider that the OF were pooled, and a possible dilution effect should be taken into account. Previous studies expressed concern about the impact of dilution and incomplete group sampling when pooling saliva from pigs voluntarily chewing sampling ropes. When pooled samples are collected from a group of animals, the contribution of individual animals to the pool is unknown and, therefore, is suitable only to evaluate the current group status. Positive pools indicate that at least one individual sample within the pool is positive; therefore, it is necessary to retest each sample to decode the positive from the negative animals. On the contrary, it is possible that positive animals may not be included in the pool, leading to seropositive subjects being misdiagnosed by reducing the value below the cut-off point [23]. Modifications to the test protocol (e.g., increasing the test volumes, sample dilutions, incubation times, temperature, kit reagents and cut-off point) may be necessary to optimise the performance of the assay [19]. These modifications need to be evaluated and validated against the current Gold Standard (blood serum samples) for assessing the sensitivity and specificity of 
the assay. At present, only the MJ has been validated and accepted as a surveillance option for the detection of anti-Salmonella antibodies [41]. However, considering the cost-effective benefit of using the OF for diagnostic purposes, at farm level, a lower sensitivity may be offset, either by collecting a higher number of samples at each sampling point or by using routine surveillance testing (every 2-4 weeks) [23].

In porcine $\mathrm{OF}$, antibody concentrations are much lower compared to serum. It was noted that IgG concentrations in OF are 800 times lower than in serum [11]. Despite the limitations of our study, such as the lack of a baseline for antibodies in the study herds and the lack of information regarding the course of infection and exposure, the results show that $\mathrm{OF}$ is a promising sample type for monitoring IgG levels in $\mathrm{V}$ farms.

The animals' behaviour in relation to age is an important factor that should be taken into account as it can affect the success of the OF sampling and the results of the study. A number of ropes were discarded because the sows did not chew them; therefore, the number of OF samples collected from sows was lower than that of the samples collected from offspring. In growing pigs, their natural exploratory behaviour facilitates the collection of $\mathrm{OF}$, whereas older animals, such as gestating sows or boars, are generally less curious and less motivated to explore materials [42,43]. For this reason, the collection of OF samples from sows is usually conducted on individually housed animals instead of on group-housed animals [42]. However, the training of sows by repeated exposure to the collection process seems to improve the animals' interest to chew the device [23].

The anti-Salmonella IgG detection in OF appears to be a promising technique, as Decorte et al. [15] already showed, and IgG in OF is the most reliable immunological isotype for monitoring specific antibody immunity in vaccinated/infected pigs.

However, the results obtained must be evaluated with caution due to some limitations of the study: in fact, the number of herds and samples examined was relatively low and no information on the serological status of the animals was available. Moreover, pigs of different ages with different health statuses and different behaviours were evaluated.

\section{Conclusions}

The current study, focusing on OF anti-Salmonella IgG, provides a preliminary indication of the potential value of this sample type on pig farms. On-farm surveillance of zoonotic diseases at the pre-harvest level is useful to help anticipate a public health problem long before it escalates. In this context, OF sampling represents a promising approach to meet this objective. The detection of specific anti-Salmonella IgG in OF could allow for the detection of Salmonella-positive batches of pigs and the adoption of specific prophylaxis measures at the slaughterhouse (e.g., end-of-day slaughter of high prevalence batches). However, further studies are needed to evaluate the correlation between Salmonella isolation and the presence of specific $\operatorname{IgG}$ in animals, in order to establish an effective monitoring protocol on farms. Further studies are necessary to confirm and expand our findings. It is, therefore, recommended that further larger scale studies are carried out in order to give greater confidence in the reliability of the method. A larger study may have enabled the results to be modeled directly against the Salmonella prevalence and count data, which might have produced more biologically relevant results.

Author Contributions: Conceptualisation, F.M., R.P.S. and A.D.L.; methodology, S.A.C., R.D. and A.D.L.; software, F.O.; validation, A.D.L. and F.O.; formal analysis, F.O. and C.B.; investigation, A.D.L. and S.A.C.; data curation, F.O.; writing-original draft preparation, A.D.L.; critical review and revising of paper, R.P.S. and R.D.; supervision, F.M. All authors have read and agreed to the published version of the manuscript.

Funding: This research was funded by the UK Department for Environment, Food and Rural Affairs under Project OZ 0344.

Institutional Review Board Statement: No ethical approval was required for the sample types collected in this study. Faecal samples were collected from the floor and the pigs voluntarily chewed the ropes that were hung in their pens. No direct procedure was carried out on the animals and, 
therefore, this work did not require ethical approval under the Animals Scientific Procedures Act 1986, which regulates this field in the UK.

Acknowledgments: The authors are grateful to Verena Goetter for the critical review of the manuscript.

Conflicts of Interest: The authors would like to state that there was no conflict of interest resulting from funding or otherwise.

\section{References}

1. De Jong Skierus, B. Human Salmonellosis-Impact of Travel and Trade from a Swedish Perspective. Ph.D. Thesis, Karolinska Institutet, Stockholm, Sweden, 2006.

2. European Food Safety Authority; European Centre for Disease Prevention and Control. The European Union One Health 2019 Zoonoses Report. EFSA J. 2021, 19, e06406. [CrossRef]

3. Wales, A.; Weaver, J.; McLaren, I.; Smith, R.; Mueller-Doblies, D.; Davies, R. Investigation of the distribution of Salmonella within an integrated pig breeding and production organisation in the United Kingdom. ISRN Vet. Sci. 2013, 2013, 943126. [CrossRef]

4. European Food Safety Authority. Scientific opinion on a quantitative microbiological risk assessment of Salmonella in slaughter and breeder pigs. EFSA J. 2010, 8, 1-90. [CrossRef]

5. Alban, L.; Stege, H.; Dahl, J. The new classification system for slaughter-pig herds in the Danish Salmonella surveillance-andcontrol program. Prev. Vet. Med. 2002, 53, 133-146. [CrossRef]

6. Alban, L.; Barfod, K.; Petersen, J.V.; Dahl, J.; Ajufo, J.C.; Sandø, G.; Krog, H.H.; Aabo, S. Description of Extended Pre-Harvest Pig Salmonella Surveillance-and-Control Programme and its Estimated Effect on Food Safety Related to Pork. Zoonoses Public Health 2010, 57, 6-15. [CrossRef]

7. Andres, V.M.; Davies, R.H. Biosecurity measures to control Salmonella and other infectious agents in pig farms: A review. Compr. Rev. Food Sci. Food Saf. 2015, 14, 317-335. [CrossRef]

8. Ramirez, A.; Wang, C.; Prickett, J.R.; Pogranichniy, R.; Yoon, K.J.; Main, R.; Johnson, J.K.; Rademacher, C.; Hoogland, M.; Hoffmann, P.; et al. Efficient surveillance of pig populations using oral fluids. Prev. Vet. Med. 2012, 104, 292-300. [CrossRef] [PubMed]

9. Dron, N.; Doyle, R.; Jover-Hernandez, M.; Holyoake, T. Detection of Actinobacillus Pleuropneumoniae in Pigs Using Pooled Oral Fluids. Available online: http:/ / porkcrc.com.au/wp-content/uploads/2014/05/121209-NDron-Honours-Thesis.pdf (accessed on 1 March 2021).

10. McKie, A.; Vyse, A.; Maple, C. Novel methods for the detection of microbial antibodies in oral fluid. Lancet Infect. Dis. 2002, 2, 18-24. [CrossRef]

11. Olsen, C.; Karriker, L.; Wang, C.; Binjawadagi, B.; Renukaradhya, G.; Kittawornrat, A.; Lizano, S.; Coetzee, J.; Main, R.; Meiszberg, A.; et al. Effect of collection material and sample processing on pig oral fluid testing results. Vet. J. 2013, 198, 158-163. [CrossRef] [PubMed]

12. Mandel, I.D. Salivary diagnosis: Promises, promises. Ann. N. Y. Acad. Sci. 1993, 694, 1-10. [CrossRef]

13. Brandtzaeg, P.; Fjellanger, I.; Gjeruldsen, S.T. Human secretory immunoglobulins. I. Salivary secretions from individuals with normal or low levels of serum immunoglobulins. Scand. J. Haematol. 1970, 12, 1-83.

14. DeBuysscher, E.V.; Berman, D.T. Secretory immune response in intestinal mucosa and salivary gland after experimental infection of pigs with transmissible gastroenteritis virus. Am. J. Vet. Res. 1980, 41, 1214-1220.

15. Decorte, I.; Van Breedam, W.; Van der Stede, Y.; Nauwynck, H.J.; De Regge, N.; Cay, A.B. Detection of total and PRRSVspecific antibodies in oral fluids collected with different rope types from PRRSV-vaccinated and experimentally infected pigs. BMC Vet. Res. 2014, 10, 134. [CrossRef]

16. Gutierrez, A.M.; Ceron, J.; Fuentes-Rubio, M.; Tecles, F.; Beeley, J.A. A proteomic approach to porcine saliva. Curr. Protein Pept. Sci. 2014, 15, 56-63. [CrossRef]

17. Prickett, J.R.; Zimmerman, J.J. The development of oral fluid-based diagnostics and applications in veterinary medicine. Anim. Health Res. Rev. 2010, 11, 207-216. [CrossRef] [PubMed]

18. Fablet, C.; Renson, P.; Pol, F.; Dorenlor, V.; Mahe, S.; Eono, F.; Eveno, E.; Le Dimna, M.; Liegard-Vanhecke, D.; Eudier, S.; et al. Oral fluid versus blood sampling in group-housed sows and finishing pigs: Feasibility and performance of antibody detection for porcine reproductive and respiratory syndrome virus (PRRSV). Vet. Microbiol. 2017, 204, 25-34. [CrossRef]

19. Cameron, S.O.; Carman, W.F. The use of the OraSure ${ }^{\circledR}$ collection device for hepatitis virus testing in health care settings. J. Clin. Virol. 2005, 34, S22-S28. [CrossRef]

20. Prickett, J.R.; Kim, W.; Simer, R.; Yoon, K.-J.; Zimmerman, J. Oral-fluid samples for surveillance of commercial growing pigs for porcine reproductive and respiratory syndrome virus and porcine circovirus type 2 infections. J. Swine Health Prod. 2008, 16, 86-91.

21. Dawson, L.L. Oral Fluid as a Non-Invasive Alternative Diagnostic Medium for Disease Monitoring in Pigs. Ph.D. Thesis, Newcastle University, Newcastle upon Tyne, UK, 2015.

22. Dietze, K.; Tucakov, A.; Engel, T.; Wirtz, S.; Depner, K.; Globig, A.; Kammerer, R.; Mouchantat, S. Rope-based oral fluid sampling for early detection of classical swine fever in domestic pigs at group level. BMC Vet. Res. 2017, 13, 5. [CrossRef] [PubMed] 
23. Henao-Diaz, A.; Gimenez-Lirola, L.; Baum, D.H.; Zimmerman, J. Guidelines for oral fluid-based surveillance of viral pathogens in swine. Porc. Health Manag. 2020, 6, 28. [CrossRef] [PubMed]

24. Smith, R.P.; Andres, V.; Martelli, F.; Gosling, B.; Marco-Jimenez, F.; Vaughan, K.; Tchorzewska, M.; Davies, R. Maternal vaccination as a Salmonella Typhimurium reduction strategy on pig farms. J. Appl. Microbiol. 2018, 124, 274-285. [CrossRef] [PubMed]

25. Martelli, F.; Gosling, R.; McLaren, I.; Wales, A.; Davies, R. Development and testing of external quality assessment samples for Salmonella detection in poultry samples. Lett. Appl. Microbiol. 2014, 59, 443-448. [CrossRef] [PubMed]

26. Grimont, P.A.; Weill, F.-X. Antigenic Formulae of the Salmonella Serovars, 9th ed.; WHO Collaborating Centre for Reference and Research on Salmonella: Geneva, Switzerland, 2007; pp. 1-166. Available online: https://www.pasteur.fr/sites/default/files/ veng_0.pdf (accessed on 1 March 2021).

27. Farzan, A.; Friendship, R.M.; Dewey, E. Evaluation of enzyme-linked immunosorbent assay (ELISA) tests and culture for determining Salmonella status of a pig herd. Epidemiol. Infect. 2007, 135, 238-244. [CrossRef] [PubMed]

28. Vico, J.P.; Mainar-Jaime, R.C. The use of meat juice or blood serum for the diagnosis of Salmonella infection in pigs and its possible implications on Salmonella control programs. J. Vet. Diagn. Investig. 2011, 23, 528-531. [CrossRef] [PubMed]

29. De Lucia, A.; Cawthraw, S.; Davies, R.; Smith, R.P.; Bianco, C.; Ostanello, F.; Martelli, F. Correlation of Anti-Salmonella Antibodies Between Serum and Saliva Samples Collected From Finisher Pigs. Front. Vet. Sci. 2020, 6, 1-7. [CrossRef]

30. Murray, A.; Lawrence, G.P. How should the repeatability of clinical measurements be analysed? An assessment of analysis techniques with data from cardiovascular autonomic function tests. QJM Int. J. Med. 1993, 86, 831-836.

31. Atkinson, B.M.; Bearson, B.L.; Loving, C.L.; Zimmerman, J.J.; Kich, J.D.; Bearson, S.M.D. Detection of Salmonella-specific antibody in swine oral fluids. Porc. Health Manag. 2019, 5, 2-5. [CrossRef]

32. Clouting, C.; Davies, R.H. Evaluation of the Salmonella meat-juice ELISA in the UK situation. In Proceedings of the Fourth International Symposium on the Epidemiology and Control of Salmonella and Other Food Borne Pathogens in Pork, Leipzig, Germany, 2-5 September 2001.

33. Brito, J.R.; Hinton, M.; Stokes, C.R.; Pearson, G.R. The humoral and cell mediated immune response of young chicks to Salmonella typhimurium and S. Kedougou. Br. Vet. J. 1993, 149, 225-234. [CrossRef]

34. Garrido, V.; Sanchez, S.; Roman, B.S.; Zabalza-Barangua, A.; Diaz-Tendero, Y.; de Frutos, C.; Mainar-Jaime, R.C.; Grillo, M.J. Simultaneous infections by different Salmonella strains in mesenteric lymph nodes of finishing pigs. BMC Vet. Res. 2014, 10, 59. [CrossRef]

35. Janeway, C.A., Jr.; Travers, P.; Walport, M.; Shlomchik, M.J. The distribution and functions of immunoglobulin isotypes. In Immunobiology: The Immune System in Health and Disease, 5th ed.; Garland Science: New York, NY, USA, 2001.

36. Roesler, U.; Heller, P.; Waldmann, K.H.; Truyen, U.; Hensel, A. Immunization of Sows in an Integrated Pig-breeding Herd using a Homologous Inactivated Salmonella Vaccine Decreases the Prevalence of Salmonella typhimurium Infection in the Offspring. Zoonoses Public Health 2006, 53, 224-228. [CrossRef] [PubMed]

37. Cawthraw, S.A.; Feldman, R.A.; Sayers, A.R.; Newell, D.G. Long-term antibody responses following human infection with Campylobacter jejuni. Clin. Exp. Immunol. 2002, 130, 101-106. [CrossRef] [PubMed]

38. Luzza, F.; Maletta, M.; Imeneo, M.; Doldo, P.; Marasco, R.; Biancone, L.; Pallone, F. Salivary specific IgG is a sensitive indicator of the humoral immune response to Helicobacter pylori. FEMS Immunol. Med. Mic. 1995, 10, 281-283. [CrossRef]

39. Van Winsen, R.; Van Nes, A.; Keuzenkamp, D.; Urlings, H.; Lipman, L.; Biesterveld, S.; Snijders, J.; Verheijden, J.; Van Knapen, F. Monitoring of transmission of Salmonella enterica serovars in pigs using bacteriological and serological detection methods. Vet. Microbiol. 2001, 80, 267-274. [CrossRef]

40. Peeters, L.; Dewulf, J.; Boyen, F.; Brossé, C.; Vandersmissen, T.; Rasschaert, G.; Heyndrickx, M.; Cargnel, M.; Pasmans, F.; Maes, D. Effects of attenuated vaccine protocols against Salmonella Typhimurium on Salmonella serology in subclinically infected pig herds. Vet. J. 2019, 249, 67-72. [CrossRef] [PubMed]

41. Mainar-Jaime, R.C.; Casanova-Higes, A.; Andrés-Barranco, S.; Vico, J.P. Looking for new approaches for the use of serology in the context of control programmes against pig salmonellosis. Zoonoses Public Health 2018, 65, e222-e228. [CrossRef]

42. Pepin, B.; Liu, F.; Main, R.; Ramirez, A.; Zimmerman, J. Collection of oral fluid from individually housed sows. J. Swine Health Prod. 2015, 23, 35-37.

43. Kittawornrat, A.; Prickett, J.; Chittick, W.; Wang, C.; Engle, M.; Johnson, J.; Patnayak, D.; Schwartz, T.; Whitney, D.; Olsen, C.; et al. Porcine reproductive and respiratory syndrome virus (PRRSV) in serum and oral fluid samples from individual boars: Will oral fluid replace serum for PRRSV surveillance? Virus Res. 2010, 154, 170-176. [CrossRef] [PubMed] 